\title{
氯酸钾对花生生长的毒害效应
}

\author{
黎华寿 ${ }^{1}$ 张修玉 $^{1} \quad$ 曾祥有 ${ }^{2}$ 聂呈荣 $^{1}$ \\ (1 华南农业大学热带亚热带生态研究所,广州 510642) \\ （2 广东省茂名市水果科学研究所, 广东茂名 525000）
}

\begin{abstract}
摘 要 氯酸盐是一类毒性强的氧化剂, 曾被作为非选择性除草剂和脱叶剂大量施用,近年来, 氯酸钾作为产期调 控剂也在龙眼 (Dimocarpus longan) 反季节生产中大量应用。然而, 氯酸根离子强氧化性对生物体有明显的毒害效 应, 其残留及次生污染物对水体和土壤环境也存在较强的污染效应。龙眼园通常间种花生(Arachis hypogaea), 该文 实验研究了氯酸钾和氯化钠对花生生长的毒害效应。结果表明, 当浸种溶液的 $\mathrm{KClO}_{3}$ 浓度高于 $50 \mathrm{mg} \cdot \mathrm{L}^{-1}$ 时, 花生 种子的发芽率和胚根长度大大降低, 幼芽的电解质渗漏率和过氧化氢酶活性显著升高; 当土壤中 $\mathrm{KClO}_{3}$ 浓度高于 $50 \mathrm{mg} \cdot \mathrm{kg}^{-1}$ 时, 会使花生幼苗叶片质膜透性增大, 而硝酸还原酶活性、叶绿素含量和根系活力显著降低, 氯酸钾的毒 害效应远远超过氯化钠的盐害效应, 高于 $50 \mathrm{mg} \cdot \mathrm{kg}^{-1}$ 的 $\mathrm{KClO}_{3}$ 还能使花生荚果期植株的光合速率、蒸腾速率、叶绿 素含量、根系活力、生物量合成和根瘤菌的数量显著降低。结论是, 土壤中的 $\mathrm{KClO}_{3}$ 浓度高于 $50 \mathrm{mg} \cdot \mathrm{kg}^{-1}$ 时, 即会显 著影响花生植株的正常生长。
\end{abstract}

关键词 花生 作物 氯酸钾 毒害效应 污染生态

\section{TOXIC EFFECTS OF POTASSIUM CHLORATE ON PEANUT GROWTH}

\author{
LI Hua-Shou ${ }^{1}$ ZHANG Xiu-Yu ${ }^{1}$ ZENG Xiang-You ${ }^{2}$ and NIE Cheng-Rong ${ }^{1}$ \\ (1 Institute of Tropical and Subtropical Ecology, South China Agricultural University, Guangzhou 510642, China)
}

(2 Fruit Institute of Maoming City, Maoming, Guangzhou 525000, China)

\begin{abstract}
Chlorates are a group of oxidizers with strong toxic effects that can cause significant damage to plants. However, chlorates have been used as a non-selective herbicide, defoliant and desiccant. It was found that potassium chlorate can induce flowering in longan (Dimocarpus longan). It has been widely used in longan orchards in recent years, especially in north Thailand and south China, but research has shown that potassium chlorate is toxic to organisms. It can also contaminate soil and water. The aim of this study was to determine the effects of potassium chlorate stress on the growth and physiological response of peanut (Arachis hypogaea).

The main method used to induce flower initiation in longan for off-season production is the application of potassium chlorate under the tree canopy. Most research efforts have been devoted to determining the optimum time and amount of application. Little is known about the phytotoxic effects of potassium chlorate on peanut plants, which usually is planted within longan orchards. In this paper, the effects of potassium chlorate on the growth of peanut plants were studied. Using the soil culture method, six potassium chlorate treatments were used to assess its effects on seed germination, chlorophyll content, levels of electrolytic leakage (EL), membrane permeability (MP), and activities of nitrate reductase ( NR) and catalase (CAT) in peanut seedling leaves, as well as the biomass and number of Rhizobium, and the transpiration and photosynthetic rates in mature peanut plants. The results showed that: when the concentration of potassium chlorate was higher than 50 $\mathrm{mg} \bullet \mathrm{L}^{-1}$, the germination rates of peanut seeds and root lengths were greatly reduced, while the amount of electrolytic leakage and the activity of CAT were increased significantly. When the concentration of potassium chlorate was higher than $50 \mathrm{mg} \cdot \mathrm{kg}^{-1}$ in soil, its phytotoxic effects resulted in a significant increase in MP in seedling leaves, and a decrease in NR, chlorophyll, biomass and the number of Rhizobium, and a reduction in transpiration and photosynthesis. It can be concluded that soil concentrations of potassium chlorate higher than $50 \mathrm{mg} \bullet \mathrm{kg}^{-1}$ would have a significantly negative effect on the growth of peanut plants.
\end{abstract}

Key words Peanut (Arachis hypogaea), Crop, Potassium chlorate, Toxicity effects, Pollution ecology 
氯酸盐是植物必需微量元素氯在环境中的存在 形式之一, 但氯酸根离子强氧化性却对许多植物具 有明显的毒性。文献记载, 除苔藓外, 氯酸盐对绿色 植物生长有 3 到 6 个月的持续毒害作用, 其毒性不 但对牵牛花 (Pharbitis nil)、加拿大蓟 (Cirsium arvense)和詹森草( Sorghum halepense)等杂草具有良好 的控制效果，而且对棉花 (Gossypium)、玉秘蜀 ( Zea mays)、亚麻( Linum tenuifolium) 和大豆( Glycine max) 等农作物具有脱叶功能, 对微生物也有毒害作用, 因 此氯酸盐曾被作为非选择性除草剂、脱叶剂和土壤 消毒剂而大量施用 (Crafts \& Robbins，1962; Klingman \& Ashton, 1975; Agaev et al., 1986)。同时,由于工 业上大量使用 $\mathrm{ClO}_{2}$ 和 $\mathrm{Cl}_{2}$ 进行纸浆漂白、自来水消 毒、水产养殖以及食品保鲜和废水废气处理等, 其过 程中产生的副产物 (Disinfection by-products, DBPs) 也 含有大量的 (高/亚) 氯酸根离子 (Siddiqui et al., 1993; Veschetti et al., 2005); 另外, 含(高)氯酸钾的 炸药、烟火和火箭推进器燃料中的 (高) 氯酸根离子 也最终归趋于土壤和水环境 (Logan, 2001), 这无疑 给农业生态环境和人类健康带来潜在威胁。为此, $\mathrm{ClO}_{4}{ }^{-} 、 \mathrm{ClO}_{3}{ }^{-}$和 $\mathrm{ClO}_{2}{ }^{-}$都包括在美国联邦法规饮用 水优先控制的消毒副产物目录中, 美国环保署 (USEPA) 推荐在出厂水中 $\mathrm{ClO}_{2} 、 \mathrm{ClO}_{3}{ }^{-}$和 $\mathrm{ClO}_{2}{ }^{-}$总剩 余浓度不得大于 $10 \mathrm{mg} \cdot \mathrm{L}^{-1}$, 世界卫生组织建议 (高)氯酸根在饮用水中的含量应减到尽可能少 （USEPA，2002a，2000b; WHO，2005）。哥伦比亚环 保部 (MEBC，2005)对环境水质中的氯酸盐限制标 准(建议) 为: 饮用水源 $2.4 \mathrm{mg} \cdot \mathrm{L}^{-1}$, 畜牧饮用水 3 $\mathrm{mg} \bullet \mathrm{L}^{-1}$, 海水养殖用水 $5 \mathrm{mg} \cdot \mathrm{L}^{-1}$, 淡水养殖用水 30 $\mathrm{mg} \cdot \mathrm{L}^{-1}$ 。

当前, 氯酸钾大量应用于龙眼 (Dimocarpus longan )产期调控是其进入环境的另一条重要途径。自 从 1998 年台湾学者颜昌瑞等 (1998) 利用氯酸钾作 为植物生长调节剂给龙眼催花获得成功以来, 我国 福建、广西、广东、海南等龙眼主产区以及泰国等东 南亚国家和美国夏威夷及佛罗里达也开展了试验和 推广研究,均获得良好效果(陈美媛等, 2001; 贺海 英和王泽槐，2001；柯冠武，2001; 温永盟，2001; Subhadrabandhu \& Yapwattanaphun, 2001), 经过几年 的试验探索, 龙眼产期化学调控技术不断成熟和推 广应用 (李建光等, 2003; 韩剑等, 2003; 曾祥有等, 2004; Manochai et al., 2005)。此外, 氯酸钾对热带 果树芒果 (Mangifera indica) 也有一定的催花促果作 用 (Subhadrabandhu \& Yapwattanaphun, 2001)。应用
氯酸钾调控龙眼产期技术, 既可对常规栽培不结果 的部分龙眼树进行催花挂果, 减少或避免“大小年” 的出现, 又可保证龙眼鲜果在不同季节特别是在节 假日均衡供应市场, 这对提高龙眼市场竞争力和产 品附加值、保证龙眼生产健康发展有重要意义 (Huang et al., 2005; Kanaree \& Pankasemsuk, 2005), 近年泰国以氯酸钾处理的龙眼产量约占全年总产的 $40 \%$, 占出口外销龙眼一半以上 (Anupunt \& Sukhvibul, 2005)。广东茂名市近 5 年来推广氯酸钾 生产反季节龙眼面积近 $6667 \mathrm{hm}^{2}$, 增加经济效益近 千万元。但是, 随着氯酸钾对果树花期调控技术的 推广, 施用氯酸钾的果园日渐增多, 氯酸根离子毒性 及其残留次生污染对农业生态环境产生的消极影响 越来越受到关注, 其生态风险不容忽视。

目前,有关氯酸盐对植物的毒害机理还研究不 多。已有研究证明, 氯酸根离子在常温下对藻类 ( Rosemarin et al., 1986, 1990, 1994; Wuk \& Hutchinson, 1995; Dolf et al ., 1998; Stauber, 1998)、 小麦( Triticum aestivum) (Aberg, 1947)、番茄( Lycopersicon eseulentum) (Hofstra，1977) 和水稻( Oryza sativa) (Borges et al.，2004) 有明显的浓度梯度毒害效应。 氯酸钾每分子含有 3 个氧, 且 $\mathrm{ClO}_{3}{ }^{-}$的结构是 $\mathrm{SP}^{3}$ 杂 化类型, 导致其具有强氧化性。A Aberg ( 1947) 首先提 出氯酸根离子本身对植物细胞无毒, 但转化为亚氯 酸根和次氯酸根后就表现出毒性, 同时植物根系对 $\mathrm{ClO}_{3}{ }^{-}$和 $\mathrm{NO}_{3}{ }^{-}$的吸收机制是一致的, 氯酸根通过与 硝酸盐竞争硝酸根载体而被吸收; 其后也有许多研 究证实, 植物吸收 $\mathrm{ClO}_{3}{ }^{-}$, 会抑制植物细胞对 $\mathrm{NO}_{3}{ }^{-}$ 的吸收和运输, 导致植物缺氮进而影响植物体的生 理、营养和生殖生长 (Solomonsson \& Vennesland, 1972；Balch，1987）。也有研究证明, $\mathrm{ClO}_{3}$ - 被植物根 系吸收并输送到植物各器官后, 其强氧化性对植物 有直接的毒害效应(Borges et al ., 2004), 并且 $\mathrm{ClO}_{3}{ }^{-}$ 及其转化的 $\mathrm{ClO}_{2}{ }^{-}$在植物体内的积累, 会增加植物 体内过氧化氢酶的含量, 提高植物细胞的呼吸效率 (Klingman \& Ashton, 1975), 降低植物对碳水化合物 的合成, 致使叶片提前脱落, 植物过早衰老 (Wright \& Sprenkel, 1996; Brown, 1997)。但也有研究证实, 部 分微生物和植物中硝酸还原酶缺失的突变体如拟南 芥突变体等对氯酸盐具有免疫能力, 可以利用氯酸 盐作为篮选这些突变体生物的有效工具 ( Singh et al., 1977; Doddema et al., 1978)。同时, 研究发现 有些厌氧细菌拥有一套与一般生物不同的氯酸根代 谢系统, 可借助氯酸还原酶将 $\mathrm{ClO}_{3}{ }^{-}$转化为 $\mathrm{ClO}_{2}{ }^{-}$, 
再由亚氯酸歧化酸 (Chlorete dismutase) 直接分解为 无毒的 $\mathrm{Cl}^{-}$, 因此这些微生物是环境中降解 $\mathrm{ClO}_{3}{ }^{-}$的 主力军和“生态工具”1) (Wallace et al ., 1996; Logan, 1998; Xu et al., 2004; Steinberg et al ., 2005)。但类 似的代谢途径是否在动物和植物中存在, 仍有待进 一步研究。

此外, 目前有关氯酸盐对豆科作物的影响研究 还少见报道。花生(Arachis hypogaea) 是我国主要经 济作物, 在我国南方部分龙眼果园等也有套种花生、 大豆的习惯。施用氯酸钾和其它污染途径带来的氯 酸根离子, 很有可能会对花生等作物产生影响, 如国 内生产氯酸钾的主要厂家之一的广西贺州光明化工 厂, 就曾出现农民与厂家发生氯酸钾污染水稻、花生 的污染纠纷。此外, 由于花生具有根瘤菌, 这与龙眼 菌根有相似之处, 同时已有研究证明植物根系大都 不能选择氯酸根与硝酸盐, 两者的竞争是否会在固 氮根瘤上有所体现仍有待研究, 为此我们选择花生 作为材料, 研究氯酸钾及其残留对花生生长的影响, 旨在为明确氯酸盐对豆科作物的生态毒理提供参 考。

\section{1 材料和方法}

\section{1 供试材料}

花生品种选用生产上应用较广的“粤油 19’, 盆 栽供试土壤采用华南农业大学农学院试验田 $0 \sim 20$ $\mathrm{cm}$ 土层的粘壤土, $\mathrm{pH}$ 值 5.48 , 全氮 $0.92 \mathrm{~g}^{\bullet} \mathrm{kg}^{-1}$, 速 效氮 $112.32 \mathrm{mg} \bullet \mathrm{kg}^{-1}$, 全磷 $1.15 \mathrm{~g} \bullet \mathrm{kg}^{-1}$, 速效磷 $48.32 \mathrm{mg} \bullet \mathrm{kg}^{-1}$, 全钾 $22.20 \mathrm{~g} \bullet \mathrm{kg}^{-1}$, 速效钾 126.65 $\mathrm{mg}^{\bullet} \mathrm{kg}^{-1}$, 有机质 $16.30 \mathrm{~g} \bullet \mathrm{kg}^{-1}$, EC 值 $0.25 \mathrm{mS} \bullet$ $\mathrm{cm}^{-1}, \mathrm{Cl}^{-}$为 $0.033 \mathrm{mg} \bullet \mathrm{kg}^{-1}$, 主要重金属指标符合国 家土壤环境质量标准。

所用 $\mathrm{KClO}_{3}$ 试剂为市售化学纯, 幼苗光照培养 室培养时使用去离子水,盆栽使用双蒸水。

\section{2 实验设计}

为区别氯酸钾强氧化性与盐害对花生萌发和幼 苗生长的毒害效应, 花生种子萌发和苗期的实验同 时以同浓度的氯化钠为对照实验。谭允阳等(1995) 研究表明, $\mathrm{Cl}^{-1}$ 对花生植株的胁迫临界浓度在 500 1000 之间, 所以氯酸钾强氧化性对花生植株的毒害 效应不设氯化钠的对照实验。

\subsection{1 种子发芽实验}

将 $\mathrm{KClO}_{3}$ 和 $\mathrm{NaCl}$ 分别用去离子水 (Milli-Q water) 配成 $0 、 10 、 20 、 50 、 100 、 200 \mathrm{mg} \cdot \mathrm{L}^{-1}$ 的浓度梯度处 理, 每个处理设置 4 个重复, 将每个重复的 25 粒花 生种子培养在直径 $15 \mathrm{~cm}$ 的培养血中, 上下均为相 同浓度的试剂浸泡过的双层滤纸, 然后把培养血置 于 $25{ }^{\circ} \mathrm{C}$ 的培养箱中, 保持培养血湿润, 进行发芽试 验。5 d 后测量花生种子的发芽率、胚根长、肧芽过 氧化氢酶活性和胚根研磨前后其渗漏液的电导率。

\subsection{2 幼苗生长实验}

将花生种子用双蒸水浸泡 $24 \mathrm{~h}$ 后, 选择饱满且 大小均匀一致的种子置于 $25{ }^{\circ} \mathrm{C}$ 的黑暗培养箱中培 养 $48 \mathrm{~h}$, 选择发芽均匀的种苗各 3 棵, 种在盛有供试 土壤 $1 \mathrm{~kg}$, 盆高 $15 \mathrm{~cm}$ 、口径 $15 \mathrm{~cm}$ 、盆底有排水孔和 托盆的陶瓷盆中, 置于温度 $25{ }^{\circ} \mathrm{C}$, 光照强度 12000 Lux, 空气相对湿度约 $85 \%$ 的光照培养箱进行培养, 定期加等量去离子水保持土壤湿润。幼苗预培养生 长 $15 \mathrm{~d}$ 后, 分别向各盆土壤中施加溶解有 $\mathrm{KClO}_{3}$ 或 $\mathrm{NaCl}$ 各 0、10、20、50、100、200 mg 的溶液, 各设 6 个处 理, 每处理 3 个重复, 加药 $5 \mathrm{~d}$ 后测量幼苗叶片质膜 透性、硝酸还原酶、叶绿素和根系活力。

\subsection{3 荚果期植株生长实验}

将花生种子用双蒸水浸泡 $24 \mathrm{~h}$ 后, 选择饱满且 大小均匀一致的种子置于 $25{ }^{\circ} \mathrm{C}$ 的黑暗培养箱中培 养 $48 \mathrm{~h}$, 选择发芽均匀的种苗各 3 棵, 种在盆高 18 $\mathrm{cm}$ 、口径 $25 \mathrm{~cm}$ 、盆底有排水孔的陶瓷盆中 (陶瓷盆 置于朝阳屋檐下, 遮雨不遮光), 各盆装有供试土壤 $5 \mathrm{~kg}$, 定期浇等量双蒸水, 保持土壤湿润。一个月后 一次性施加 $\mathrm{KClO}_{3}$ 处理, 各处理的土壤施加的 $\mathrm{KClO}_{3}$ 浓度分别为 $0 、 50 、 100 、 200 、 400 、 800 \mathrm{mg} \bullet \mathrm{kg}^{-1}$, 共设 6 个处理 3 个重复。两个月后测定植株的光合速率、 叶绿素含量和根系活力。 4 个月收获后测定植株的 生物量和根瘤菌数量。

\section{3 实验方法}

胚根电解质渗漏率: 各处理称 $0.2 \mathrm{~g}$ 胚根, 用 20 $\mathrm{ml}$ 去离子水浸泡 $3 \mathrm{~h}$ 后, 用 DDS-11 型电导仪测量研 磨前后溶液的电导度, 以相对电导度(研磨前后电导 度比值) 表示胚根电解质渗漏率。叶片质膜透性: 各 处理称取 $0.5 \mathrm{~g}$ 花生苗期顶部叶片用 $20 \mathrm{ml}$ 去离子 水浸泡 $3 \mathrm{~h}$ 后, 用 DDS-11 型电导仪测出浸出液在者 沸前后的电导度, 计算相对电导(研磨前后电导度比 值)来表示幼苗叶片质膜透性。 
酶液制备: 分别取胚根 $0.25 \mathrm{~g}$ 与苗期叶片 $0.5 \mathrm{~g}$ 加入 $2.5 \mathrm{ml} 0.05 \mathrm{~mol} \cdot \mathrm{L}^{-1}$ 磷酸缓冲液 $(\mathrm{pH}=7.2$ ) ( 内 含 $5 \%$ 聚乙烯基吡咯烷酮 PVP) 及少量石英砂, 冰浴 下研磨成匀浆, $0 \sim 4{ }^{\circ} \mathrm{C}, 20000 \times g$ 离心 $20 \mathrm{~min}$, 取 上层清液用于酶活性测定。

过氧化氢酶活性: 用 $0.05 \mathrm{~mol} \cdot \mathrm{L}^{-1}$ 磷酸缓冲液 $(\mathrm{pH}=7.2)$ 把 $0.6 \mathrm{ml} 30 \%$ 的 $\mathrm{H}_{2} \mathrm{O}_{2}$ 稀释至 $100 \mathrm{ml}$ 作 为底物, 在比色杯中依次加入 $1 \mathrm{ml}$ 底物、 $1.9 \mathrm{ml} 0.05$ $\mathrm{mol} \cdot \mathrm{L}^{-1}$ 磷酸缓冲液、100 $\mu \mathrm{l}$ 酶液后迅速用封口膜封 口摇匀, 立即放入分光光度计内在 $240 \mathrm{~nm}$ 波长下测 定 OD240 值, 每 $30 \mathrm{~s}$ 读数一次, 连续读 6 次, 过氧化 氢酶活性以 $\mathrm{H}_{2} \mathrm{O}_{2} \mu \mathrm{mol} \cdot \mathrm{min}^{-1} \cdot \mathrm{g}^{-1}$ 为活力单位。

硝酸还原酶活性: 采用陈薇和张德臨 (1980) 的 活体法。

叶绿素: 取主茎倒数第三叶按 Arnon( 1949)法测 定叶绿素含量。

光合速率与蒸腾速率: 在自然光照强度 1430 $1450 \mu \mathrm{mol}$ quanta $\cdot \mathrm{m}^{-2} \cdot \mathrm{s}^{-1}$, 温度 $37{ }^{\circ} \mathrm{C}$ 的上午, 用英 国 PPS 公司生产的 CIRS- II 型便携式光合作用测定 仪,测定主茎顶叶的光合作用。
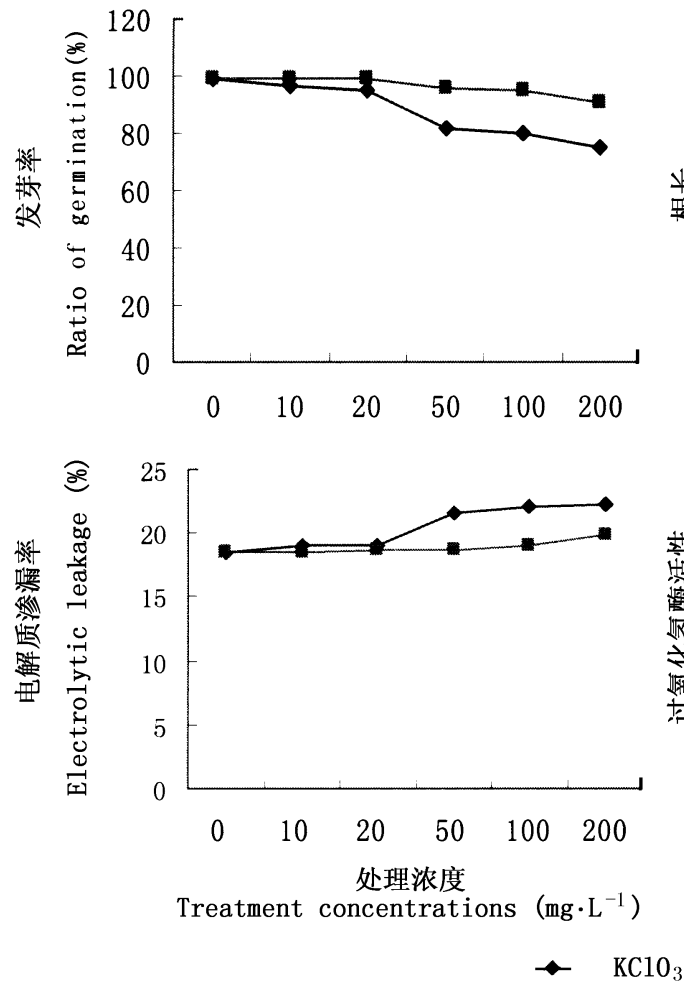

根系活力: TTC 比色法测定根系活力 (邹琦, 2000)。

\section{2 结果与分析}

\section{$2.1 \mathrm{KClO}_{3}$ 对花生种子萌发的影响}

从图 1 可见,花生种子经氯酸钾和氯化钠处理 后, 其发芽率和根长均随处理浓度的升高而降低, 电 解质渗漏率和过氧化氢酶活性则随处理浓度的升高 而升高。在 $0 \sim 200 \mathrm{mg} \cdot \mathrm{L}^{-1}$ 范围内, 经氯化钠处理的 花生种子发芽率、根长、电解质渗漏率和过氧化氢酶 活性的变化不大,均无显著差异; 而与 CK 对照, 经 氯酸钾处理的花生种子发芽率、根长、电解质渗漏率 和过氧化氢酶活性在 $50 \mathrm{mg} \cdot \mathrm{L}^{-1}$ 后变化显著, 说明 在此处理浓度范围内, 氯酸钾强氧化性对花生种子 萌发造成的毒害效应远远超过氯化钠的盐害, 且氯 酸钾浓度高于 $50 \mathrm{mg} \cdot \mathrm{L}^{-1}$ 时即会影响花生种子的正 常萌发。

\section{$2.2 \mathrm{KClO}_{3}$ 对花生苗期生长的影响}

花生幼苗经氯酸钾和氯化钠处理后, 叶片质膜 透性随处理浓度的升高而升高, 硝酸还原酶活性、叶
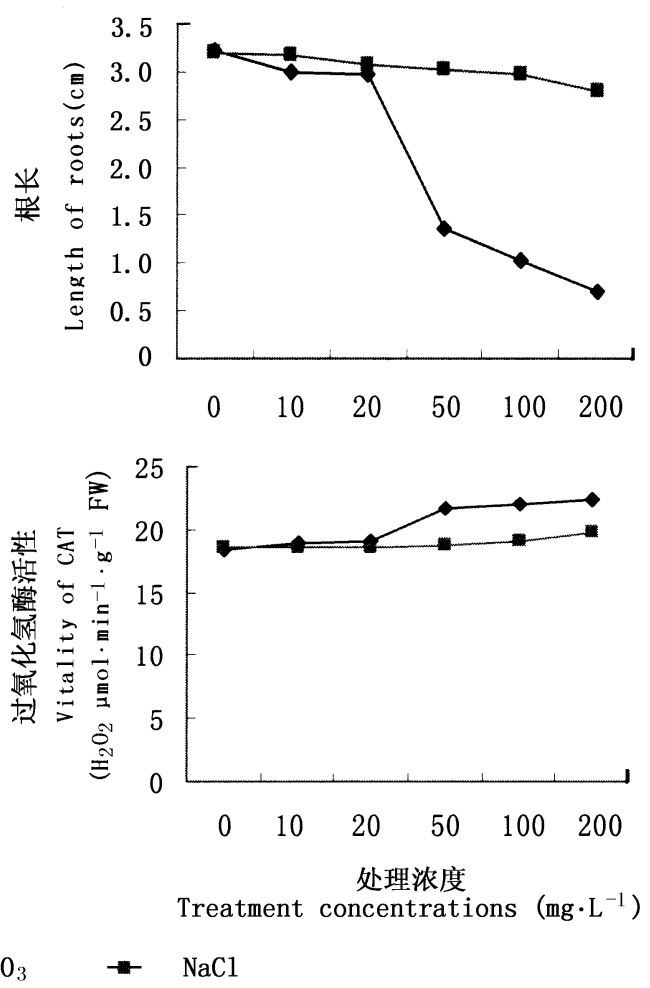
绿素含量和根系活力则随处理浓度的升高而降低 (图 2)。在 $0 \sim 200 \mathrm{mg} \bullet \mathrm{kg}^{-1}$ 范围内, 氯化钠处理的花 生幼苗叶片质膜透性、硝酸还原酶活性、叶绿素含量 和根系活力随处理浓度的升高变化不大且缓慢, 而 经氯酸钾处理过的花生幼苗叶片质膜透性、硝酸还 原酶活性、叶绿素含量和根系活力在高于 $50 \mathrm{mg}$ ・ $\mathrm{kg}^{-1}$ 后变化显著, 说明在此浓度处理范围内, 氯酸钾 强氧化性对花生幼苗的毒害效应远远高于氯化钠的 盐害效应, 且氯酸钾浓度高于 $50 \mathrm{mg} \cdot \mathrm{kg}^{-1}$ 时即会严
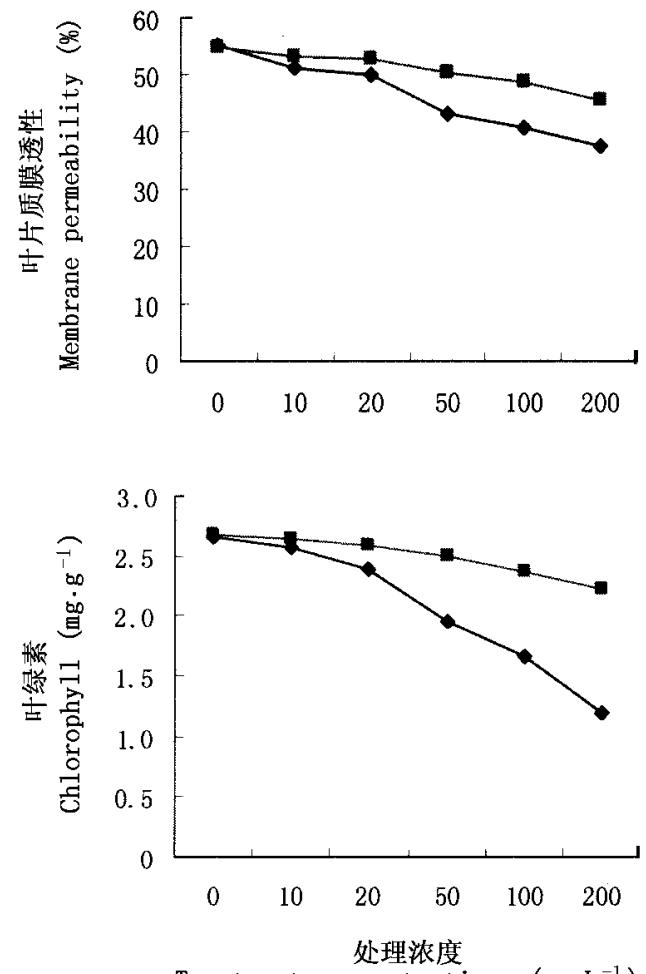

Treatment concentrations $\left(\mathrm{mg} \cdot \mathrm{L}^{-1}\right)$
重影响花生幼苗的正常生长。

\section{$2.3 \mathrm{KClO}_{3}$ 对花生荚果期植株的影响}

从表 1 可知, 花生荚果期植株的蒸腾速率、光合 速率、叶绿素含量和根系活力均随氯酸钾处理土壤 浓度的升高而降低, 且氯酸钾浓度为 $50 \mathrm{mg} \bullet \mathrm{kg}^{-1}$ 时, 花生植株的蒸腾速率、叶绿素 $\mathrm{a}$ 的含量和根系活力 与对照相比差异显著; 氯酸钾浓度达到 $100 \mathrm{mg} \cdot \mathrm{kg}^{-1}$ 时, 光合速率和叶绿素 $\mathrm{b}$ 的含量差异达到显著。

花生收获时 (表 2), 花生植株和果实的生物量
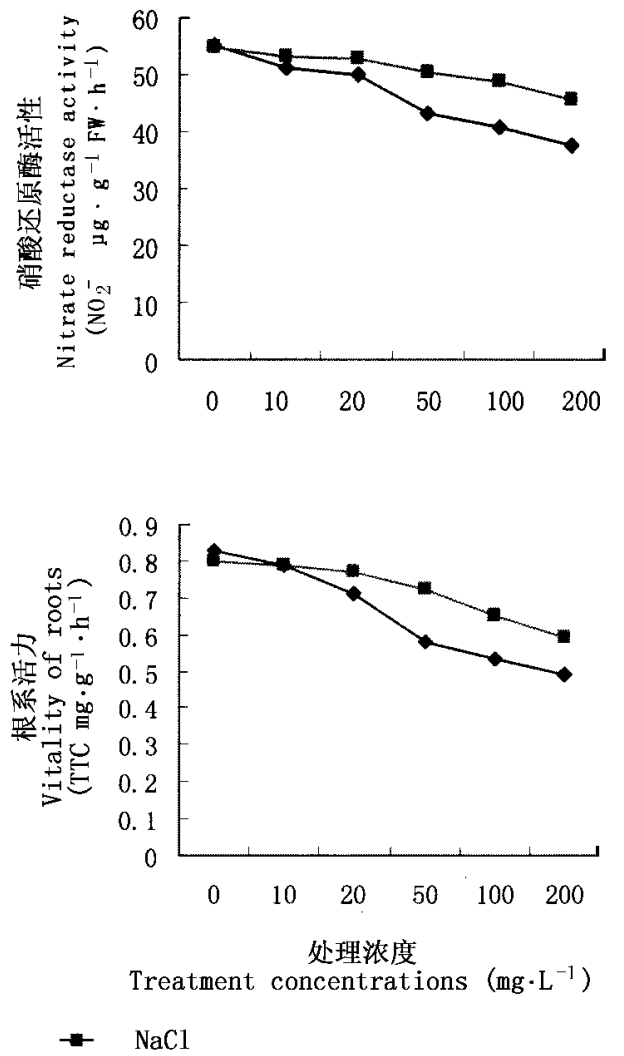

图 $2 \mathrm{KClO}_{3}$ 对花生幼苗某些生理指标的影响

Fig.2 Effects of $\mathrm{KClO}_{3}$ on some physiological index of peanut seedling

表 $1 \mathrm{KClO}_{3}$ 对花生成年植株某些生理指标的影响

Table 1 Effects of $\mathrm{KClO}_{3}$ on some physiological index of peanut plant

\begin{tabular}{cccccc}
\hline $\begin{array}{c}\mathrm{KClO}_{3} \text { 浓度 } \\
\begin{array}{c}\text { Potassium chlorate } \\
\text { concentration }\left(\mathrm{mg}^{\bullet} \mathrm{kg}^{-1}\right)\end{array}\end{array}$ & $\begin{array}{c}\text { 蒸腾速率 } \\
\text { Transpiration rate } \\
\left(\mathrm{mmol} \cdot \mathrm{m}^{-2} \cdot \mathrm{s}^{-1}\right)\end{array}$ & $\begin{array}{c}\text { 光合速率 } \\
\text { Photosynthesis rate } \\
\left(\mu \mathrm{mol} \cdot \mathrm{m}^{-2} \cdot \mathrm{s}^{-1}\right)\end{array}$ & $\begin{array}{c}\text { 叶绿素 a } \\
\text { Chlorophyll a } \\
\left(\mathrm{mg}^{\bullet} \mathrm{g}^{-1}\right)\end{array}$ & $\begin{array}{c}\text { 叶绿素 b } \\
\text { Chlorophyll b } \\
\left(\mathrm{mg}^{\bullet} \mathrm{g}^{-1}\right)\end{array}$ & $\begin{array}{c}\text { 根系活力 } \\
\text { Vitality of roots } \\
\left(\mathrm{TTC} \mathrm{g}^{\bullet} \mathrm{g}^{-1} \cdot \mathrm{h}^{-1}\right)\end{array}$ \\
\hline $\mathrm{CK}$ & $19.78 \pm 0.63^{\mathrm{a}}$ & $26.70 \pm 1.16^{\mathrm{a}}$ & $1.19 \pm 0.01^{\mathrm{a}}$ & $0.46 \pm 0.01^{\mathrm{a}}$ & $0.62 \pm 0.01^{\mathrm{a}}$ \\
50 & $16.21 \pm 0.18^{\mathrm{b}}$ & $24.85 \pm 0.55^{\mathrm{a}}$ & $1.11 \pm 0.01^{\mathrm{b}}$ & $0.44 \pm 0.01^{\mathrm{a}}$ & $0.56 \pm 0.02^{\mathrm{b}}$ \\
100 & $12.66 \pm 0.98^{\mathrm{c}}$ & $21.60 \pm 1.61^{\mathrm{b}}$ & $0.91 \pm 0.01^{\mathrm{c}}$ & $0.38 \pm 0.01^{\mathrm{b}}$ & $0.52 \pm 0.01^{\mathrm{bc}}$ \\
200 & $10.43 \pm 0.58^{\mathrm{d}}$ & $19.47 \pm 0.43^{\mathrm{bc}}$ & $0.62 \pm 0.01^{\mathrm{d}}$ & $0.23 \pm 0.01^{\mathrm{c}}$ & $0.47 \pm 0.01^{\mathrm{c}}$ \\
400 & $9.36 \pm 0.36^{\mathrm{de}}$ & $17.63 \pm 0.38^{\mathrm{c}}$ & $0.36 \pm 0.02^{\mathrm{e}}$ & $0.17 \pm 0.02^{\mathrm{d}}$ & $0.42 \pm 0.02^{\mathrm{d}}$ \\
800 & $8.48 \pm 0.28^{\mathrm{e}}$ & $16.80 \pm 0.21^{\mathrm{c}}$ & $0.27 \pm 0.02^{\mathrm{f}}$ & $0.14 \pm 0.01^{\mathrm{e}}$ & $0.35 \pm 0.03^{\mathrm{e}}$ \\
\hline
\end{tabular}


表 $2 \mathrm{KClO}_{3}$ 对花生植株生物量和根瘤菌数量的影响

Table 2 Effects of $\mathrm{KClO}_{3}$ on biomass and number of rhizobium of peanut plant

\begin{tabular}{cccccc}
\hline $\begin{array}{c}\mathrm{KClO}_{3} \text { 浓度 } \\
\begin{array}{c}\text { Potassium chlorate } \\
\text { concentration }\left(\mathrm{mg}^{\bullet} \mathrm{kg}^{-1}\right)\end{array}\end{array}$ & $\begin{array}{c}\text { 植株总鲜重 } \\
\text { Fresh weight of } \\
\text { total plant }(\mathrm{g})\end{array}$ & $\begin{array}{c}\text { 地上部鲜重 } \\
\text { Fresh weight of } \\
\text { stem (g) }\end{array}$ & $\begin{array}{c}\text { 地上部干重 } \\
\text { Dry weight of } \\
\text { stem (g) }\end{array}$ & $\begin{array}{c}\text { 荚果鲜重 } \\
\text { Fresh weight of } \\
\text { fruits }(\mathrm{g})\end{array}$ & $\begin{array}{c}\text { 根瘤菌数量(个) } \\
\text { Number of } \\
\text { Rhizobium }\end{array}$ \\
\hline $\mathrm{CK}$ & $77.30 \pm 6.37^{\mathrm{a}}$ & $46.71 \pm 5.78^{\mathrm{a}}$ & $15.21 \pm 2.08^{\mathrm{a}}$ & $27.11 \pm 2.98^{\mathrm{a}}$ & $128.25 \pm 11.00^{\mathrm{a}}$ \\
50 & $56.28 \pm 11.30^{\mathrm{ab}}$ & $42.88 \pm 9.29^{\mathrm{ab}}$ & $13.28 \pm 2.64^{\mathrm{a}}$ & $11.44 \pm 1.95^{\mathrm{b}}$ & $100.25 \pm 4.61^{\mathrm{b}}$ \\
100 & $45.53 \pm 6.08^{\mathrm{b}}$ & $28.45 \pm 5.38^{\mathrm{bc}}$ & $8.18 \pm 1.74^{\mathrm{b}}$ & $14.39 \pm 2.85^{\mathrm{b}}$ & $90.50 \pm 3.97^{\mathrm{b}}$ \\
200 & $24.30 \pm 2.82^{\mathrm{c}}$ & $17.16 \pm 2.43^{\mathrm{c}}$ & $5.98 \pm 0.57^{\mathrm{b}}$ & $4.22 \pm 1.01^{\mathrm{c}}$ & $69.00 \pm 6.42^{\mathrm{c}}$ \\
400 & $22.93 \pm 3.32^{\mathrm{c}}$ & $16.91 \pm 2.32^{\mathrm{c}}$ & $4.92 \pm 0.66^{\mathrm{b}}$ & $3.34 \pm 0.86^{\mathrm{c}}$ & $53.00 \pm 4.65^{\mathrm{cd}}$ \\
800 & $15.47 \pm 4.26^{\mathrm{c}}$ & $11.54 \pm 2.73^{\mathrm{c}}$ & $3.79 \pm 1.21^{\mathrm{b}}$ & $0.49 \pm 0.18^{\mathrm{c}}$ & $44.25 \pm 3.09^{\mathrm{d}}$ \\
\hline
\end{tabular}
0.05 )

以及根瘤菌数量均随氯酸钾浓度的升高而降低, 氯 酸钾浓度为 $50 \mathrm{mg} \cdot \mathrm{kg}^{-1}$ 时, 荚果鲜重和根瘤菌数量 与对照相比差异显著, 氯酸钾浓度达到 $100 \mathrm{mg} \cdot \mathrm{kg}^{-1}$ 时, 植株总鲜重、地上部鲜重及干重差异也达到显 著。这说明氯酸钾在土壤中的浓度高于 $50 \mathrm{mg} \cdot \mathrm{kg}^{-1}$ 时, 就会影响花生植株的正常生长和根系结瘤和结 荚果数。

\section{3 讨 论}

本实验中, 氯酸钾对花生种子萌发和幼苗生长 的影响以氯化钠为对照, 结果说明, 在本研究相同浓 度的处理范围内, 氯酸钾强氧化性对花生种子萌发 和幼苗生长的毒害远远超过氯化钠的盐害, 谭允阳 等(1995) 曾对花生植株耐氯程度研究证明, $\mathrm{Cl}^{-} \leqslant$ $500 \mathrm{mg} \cdot \mathrm{kg}^{-1}$ 时, 对花生植株生长无不良影响, 因此, 本实验的浓度设置可以排除 $\mathrm{Cl}^{-}$的盐害效应。种子 萌发过程中, 氯酸钾浓度超过 $50 \mathrm{mg} \cdot \mathrm{L}^{-1}$ 时, 其强氧 化性不但会大大降低发芽率和胚根伸长, 而且破坏 了根膜透性, 致使电解质渗漏率和过氧化氢酶活性 升高。氯酸钾在土壤中的浓度超过 $50 \mathrm{mg} \cdot \mathrm{kg}^{-1}$ 时, 不仅严重破坏幼苗叶片质膜透性, 致使幼苗期叶片 硝酸还原酶、叶绿素和根系活力均显著降低, 而且会 使成年植株的光合速率、蒸腾速率、叶绿素 $\mathrm{a}$ 和 $\mathrm{b}$ 的 含量、根系活力以及收获时植株和荚果的生物量甚 至根瘤菌数量显著下降。究其机理, 可能是氯酸钾 强氧化性破坏了硝酸还原酶等花生氮素代谢中硝态 氮转化的关键酶和硝酸还原的限速酶, 使花生在幼 苗期未能正常建立共生固氮体系，导致花生植株对 土壤无机态氮的利用受到限制, 最终影响生物量和 根瘤菌的形成。Girija 等 (2002) 研究表明, 高浓度 $\left(50 \mathrm{mmol} \cdot \mathrm{L}^{-1} \mathrm{NaCl}\right)$ 盐灌溉胁迫可导致花生幼苗脯 氨酸 (Proline) 和甜菜碱 (Glycinebetaine, GB) 持续增 加, 但氯酸钾强氧化性是否如此有待检验。
Borges 等(2004) 曾就氯酸钾在不同温度下产生 的毒性对水稻根和叶等组织细胞的生理生化和形态 解剖的毒害效应做过深入研究, 结果发现, 低温下氯 酸钾产生的毒性对水稻影响较小, 但在常温下, 氯酸 钾除增加过氧化氢酶外, 还会使叶绿体外膜消失, 降 低叶绿体的生化效率,进而影响叶片的光合速率; 同 时, 还会不同程度的损坏根毛和根系内外表皮细胞, 影响硝酸还原酶的活动。这和本试验得出的常温下 高浓度氯酸钾严重影响花生种子的萌发、幼苗和成 年植株各项生理指标的结论是一致的, 但在低温下 氯酸钾对花生毒害效应的研究还有待开展。

本试验在进行中还发现, 常温下生长的花生植 株加入氯酸钾后, 植株叶片变黄, 有落叶现象, 整个 植株生长缓慢甚至停止生长, 浓度越大, 有关受害症 状越严重, 大约 $20 \mathrm{~d}$ 后才逐渐恢复生长, 但其抗早 性大大降低, 且其后各生育期均有所延缓; 同时, 由 于氯酸钾在土壤中容易淋失和分解, 因此, 氯酸钾在 不同水分条件下对不同生育期花生生长、根瘤菌固 氮甚至土壤微生物的毒害效应也有待进一步深入探 究, 氯酸钾对花生植株各组织器官形态结构和生化 过程特别是植物-根瘤菌固氮共生体系的影响也有 待开展。

\section{参 考 文 献}

Aberg B (1947). On the mechanism of the toxic action of chlorates and some related substances upon young wheat plants. Kungl Lantbrukshogskolans Annaler, 15, 37 - 107 .

Agaev R, Danilov V, Khachaturov V, Kasymov B, Tishabaev B (1986). The toxicity to warm-blooded animals and fish of new defoliants based on sodium and magnesium chlorates. Uzbekskii Biologicheskii Zhurnal, 1, 40-43.

Anupunt P, Sukhvibul N (2005). Lychee and longan production in Thailand. Acta Horticulturae, 665, 53 - 59 .

Arnon (1949). Copper enzymes in isolated chloroplasts polyphenoloxidase in Beta vulgaris. Plant Physiology, 24, 1-15. 
Balch WM (1987). Studies of nitrate transport by marine phytoplankton using ${ }^{36} \mathrm{Cl}_{-} \mathrm{ClO}_{3}{ }^{-}$as a transport analogue. Journal of Phycology, 23, $107-118$.

Borges R, Miguel EC, Janice MR (2004). Ultrastructural, physiological and biochemical analyses of chlorate toxicity on rice seedlings. Plant Science, 166, 1057 - 1062.

Brown KM (1997). Ethylene and abscission. Physiology Plantarum, 100, $567-576$.

Chen MN (陈美暖), Zhou JS (周进水), Su GC (苏国存) (2001). Research on controlling technique in longan producing season. South China Fruit Tree (中国南方果树), 30(4), 27. (in Chinese with English abstract)

Chen W (陈薇), Zhang DY (张德䝠) (1980). The extraction, determination and purification of nitrate reductase in plant tissue. Plant Physiology Communications (植物生理学通讯), 4, 45 - 49. (in Chinese with English abstract)

Crafts AS, Robbins WW (1962). Weed Control. McGraw-Hill Press, New York, USA.

Doddema H, Hofstra JJ, Feenstra WJ (1978). Uptake of nitrate by mutants of Arabidopsis thaliana, disturbed in uptake or reduction of nitrate. I. Effect of nitrogen source during growth on uptake of nitrate and chlotate. Physiologia Plantarum, 443, 343-350.

Dolf JVW, Sander GM, Garttener CM (1998). Toxicity of chlorate and chlorite to selected species of algae, bacteria, and fungi. $E$ cotoxicology and Environmental Safety, 40, 206-211.

Girija C, Smith BN, Swamy PM (2002). Interactive effects of sodium chloride and calcium chloride on the accumulation of proline and glycinebetaine in peanut (Arachis hypogaea L.). Environmental and Experimental Botany, 47, 1 - 10 .

Han J (韩剑), Lin S (林盛), Hua M (华敏) (2003). Development of flower forcing techniques for longan (Dimocarpus longan Lour). Chinese Journal of Tropical Crops (热带作物学报), 24 (2) , 19-24. (in Chinese with English abstract)

He HY (贺海英), Wang ZH (王泽槐) (2001). Studying of highly oxidizing substances on longan blossom. Guangdong Horticulture (广东园艺) , 2(4), 42-44. (in Chinese with English abstract)

Hofstra JJ (1977) . Chlorate toxicity and nitrate reductase activity in tomato plants. Physiological Plantarum, 41, 65-69.

Huang X, Huang HB, Zeng L (2005). Lychee and longan production in China. Acta Horticulturae, 665, $27-36$.

Kanaree W, Pankasemsuk T (2005). Effect of potassium chlorate on fruit quality of 'Do' longan. Acta Horticulturae, 665, 281 283.

Ke GW (柯冠武) (2001). Practice and inspire of introducing the technique of urging longan blossom. Guangdong Horticulture (广 东园艺), 2(1), 37-38. (in Chinese with English abstract)

Klingman GC, Ashton FM (1975). Weed Science. Wiley Press, New York, USA.

Li JG (李建光), Pan XW (潘学文), Li R (李荣) (2003). Advances in research of inducing flowering by potassium chlorate ap- plication. Journal of Fruit Sinica (果树学报), 20, 410-414. (in Chinese with English abstract)

Logan BE (2001). Assessing the outlook for perchlorate remediation. Environmental Science \& Technology, 35, 482A - 487A.

Logan BE (1998). A review of chlorate and perchlorate respiring microorganisms. Bioremediation Journal, 2, 69-79.

Manochai P, Sruamsiri P, Wiriya-Alongkorn W, Naphron D, Hegele M, Bangerth F (2005). Year around off season flower induction in longan (Dimocarpus longan Lour.) trees by $\mathrm{KClO}_{3}$ applications: potentials and problems. Scientia Horticulturae, 104, $379-390$.

MEBC (2005). Water quality: ambient water quality guidelines for chlorate. In: National Library of Canada Cataloguing in Publication Data. http://wlapwww. gov. bc. ca/wat/wq/BCguidelines/ chlorate/chlorate. html. Cited 25 Feb. 2002.

Rosemarin A, Lehtinen K, Notini M (1994). Effects of pulp mill chlorate on Baltic sea algae. Environmental Pollution, 85, 3 13.

Rosemarin A, Mattsson J, Lehtinen KJ (1986) . Effects of pulp mill chlorate on Fucus vesiculosus : a summary of projects. Ophelia, 4, $219-224$.

Rosemarin A, Mattsson J, Lehtinen KJ (1990) . Effects of treated and untreated softwood pulp mill effluents on Baltic sea algae and invertebrates in model ecosystems. Nordic Pulp \& Paper Research Journal, 2, 83-87.

Siddiqui RA, Warnecke-Eberz U, Hengsberger A (1993). Structure and function of a periplasmic nitrate reductase in Alcaligenes eutrophus H16. Journal of Bacteriology, 175, 5867 - 5876.

Singh HN, Sonie KC, Singh HR (1977). Nitrate regulation of heterocyst differentiation and nitrogen fixation in a chlorate-resistant mutant of the blue-green alga Nostoc muscorum. Mutation Research, 42, $447-452$.

Solomonsson LP, Vennesland B (1972). Nitrate reductase and chlorate toxicity in Chlorella vulgaris Beijerinck. Plant Physiolo$g y, 50,421-424$.

Stauber JL ( 1998 ). Toxicity of chlorate to marine microalgae. Aquatic Toxicology, 41, 213 - 227.

Steinberg L, Trimble J, Logan BE (2005) . Enzymes responsible for chlorate reduction by Pseudomonas sp. are different from those used for perchlorate reduction by Azospira sp. FEMS Microbiology Letters, 247, $153-159$.

Subhadrabandhu S, Yapwattanaphun C (2001). Regulation off-season flowering of longan in Thailand. Acta Horticulturae, 558, $193-198$.

Tan YY (谭允阳), Ke YS (柯玉诗), Huang SQ (黄盛球) (1995). Study on enduring chlorine of peanut and the main direction of chlorine. Soil and Fertilizer (土壤肥料), 3, 33 36. (in Chinese with English abstract)

USEPA (2002a). Perchlorate environmental contamination: toxicological review and risk characterization. External review draft. NCEA-1-0503. National Center for Environmental Assessment, 
Office of Research and Development, U.S. Environmental Protection Agency, Washington, DC. http://cfpub. epa.gov/ncea/ $\mathrm{cfm} /$ recordisplay. $\mathrm{cfm}$ ? deid $=24002$. Cited 23 Aug. 2004 .

USEPA (2002b) . Report on the peer review of the U.S. Environmental Protection Agency's Draft External Review Document "Perchlorate environmental contamination: toxicological review and risk characterization". EPA/635/R02/003. National Cencter for Environmental Assessment, Office of Research and Development, U.S. Environmental Protection Agency, Washington, DC. http://www. epa. gov/ncea/pdfs/perchlorate/final-rpt. pdf. Cited 23 Aug. 2004.

Veschetti E, Cittadini B, Maresca D (2005) . Inorganic by-products in waters disinfected with chlorine dioxide. Microchemical Journal, 79, $165-170$.

Wallace W, Ward T, Breen A, Attaway H (1996). Identification of an anaerobic bacterium which reduces perchlorate and chlorate as Wolinella succinogenes. Journal of Industrial Microbiology \& Biotechnology, 16, 68-72.

Wen YM (温永盟) (2001). Approach on controlling technique in longan producing season. Guangdong Horticulture (广东园艺), 2(1), 37 - 38. (in Chinese with English abstract)

WHO (2005) . Chlorite and chlorate in drinking-water. Background document for development of $\mathrm{WHO}$ guidelines for drinking-water quality. http://www. who. int/water-sanitation-health/dwq/ chemicals/chloritechlorate/en/. Cited 5 May 2005.
Wright DL, Sprenkel RK (1996). Defoliating Cotton, Department of Agronomy. Florida Cooperative Extension Service, Institute of Food and Agricultural Sciences, University of Florida. Publication SS-AGR-21.

Wuk VDJ, Hutchinson TH (1995). The ecotoxicity of chlorate to aquatic organisms: a critical review. Ecotoxicology and Environmental Safety, 32, $244-253$.

Xu J, Trimble JJ, Steinberg L, Logan BE (2004). Chlorate and nitrate reduction pathways are separately induced in the perchlorate-respiring bacterium Dechlorosoma sp. KJ and the chloraterespiring bacterium Pseudomonas sp. PDA. Water Research, 38, $673-680$.

Yan CR(颜昌瑞), Zhao ZN (赵政男), Zhang ZW (张哲玮) (1998). Effects of chemical on introducing blossom of longan. Journal of Chinese Social Horticulture Science, (中国园艺 (台 湾) ), 44, 517 - 518. (in Chinese with English summary)

Zeng XY (曾祥有), Li HS (黎华寿), Lu HM (陆宏谋), Zeng YY (曾运友) (2004). Effects of $\mathrm{KClO}_{3}$ on flowering and fruiting of longan. Chinese Journal of Applied and Environmental Biology (应用与环境生物学报), 10,573-576. (in Chinese with English abstract)

Zou Q (邹琦) (2000). Experimental Guidance for Plant Physiolo$g y$ (植物生理学实验指导). China Agriculture Press, Beijing. (in Chinese) 